\title{
Intervenção Coronária Percutânea em Idosos: Impacto da Faixa Etária Mais Avançada ( $\geq 80$ Anos) no Perfil Clínico e nos Resultados Imediatos
}

\author{
Roberto Tadeu M. Kroll ${ }^{1}$, Luiz Fernando Tanajura ${ }^{1}$, Dimytri Alexandre A. Siqueira ${ }^{1}$, Alexandre Abizaid ${ }^{1}$, \\ Fausto Feres ${ }^{1}$, Danilo Ribeiro Galantini ${ }^{1}$, Antonio Thomaz de Andrade ${ }^{1}$, Lilian Soares Silva ${ }^{1}$, \\ Marcela Freire Buffon ${ }^{1}$, Samir Duarte Ibrahim ${ }^{1}$, Amanda G. M. R. Sousa ${ }^{1}$, J. Eduardo Sousa ${ }^{1}$
}

\section{RESU M 0}

Introdução: Com o aumento da longevidade observado nas últimas décadas, as intervenções coronárias percutâneas (ICPs) em octogenários são cada vez mais indicadas, podendo, no entanto, não apresentar os mesmos resultados de pacientes idosos com idade menos avançada. Este estudo comparou os perfis e os resultados imediatos em pacientes idosos com idade $\geq 80$ anos e $<80$ anos, procurando respostas para esses questionamentos. Método: Estudo de coorte, retrospectivo, que envolveu todos os 998 pacientes idosos tratados no triênio 2008-2010, no Instituto Dante Pazzanese de Cardiologia, divididos em dois grupos: grupo A, 192 (19,2\%) octogenários; e grupo B, 806 (81,8\%) pacientes idosos com idades entre 70 anos e 79 anos. Foram incluídos pacientes apresentando quadros clínico e angiográfico com indicação para ICP, de acordo com as diretrizes vigentes. Resultados: Os pacientes do grupo A apresentaram predomínio significante de insuficiência renal crônica $(78,6 \%$ vs. $54,8 \% ; P<0,01)$ e tenderam a apresentar mais síndromes coronárias agudas $(33,3 \%$ vs. $26,6 \% ; P=0,07)$. As lesões-alvo tipo B2/C também predominaram no grupo A $(54,8 \%$ vs. $41,2 \% ; P<0,01)$, porém, a despeito disso, esses pacientes receberam menos stents farmacológicos $(18,1 \%$ vs. $31,2 \% ; P<0,01)$. Não foi observada diferença na mortalidade ( 0 vs. $0,2 \%$; $P=0,83$ ) ou na ocorrência de infarto relacionado ao procedimento $(5,7 \%$ vs. $3,3 \% ; P=0,18)$. N ão houve necessidade da realização de cirurgia de revascularização de urgência em nenhum paciente. Conclusões: Os octogenários, que atualmente correspondem a cerca de $20 \%$ dos pacientes tratados por ICP, apresentaram maior complexidade clínica e angiográfica, receberam menos stents com liberação de medicamentos e apresentaram resultados hospitalares semelhantes aos dos menos idosos.

DESCRITO RES: Angioplastia. Stents farmacológicos. Doença das coronárias. Idoso. Idoso de 80 anos ou mais.

\author{
ABSTRACT \\ Percutaneous Coronary Intervention in the Elderly: \\ Impact of Advanced Age ( $\geq 80$ Years) on the \\ Clinical Profile and Immediate Results
}

Background: With the increase in longevity observed in the last decades, percutaneous coronary interventions (PCI) in octogenarians are increasingly recommended. However, they may not achieve the same results observed for younger elderly individuals. This study compared the profiles and immediate results of elderly patients $\geq 80$ and $<80$ years of age. Methods: We performed a retrospective cohort study including 998 elderly patients treated from 2008 to 2010 at the Instituto Dante Pazzanese de Cardiologia, divided into two groups: group A, 192 (19.2\%) octogenarians; and group $B, 806(81.8 \%)$ individuals with ages ranging from 70 to 79 years. Patients with clinical and angiographic indications for $\mathrm{PCl}$ were included according to the current guidelines. Results: Group A patients had a significant prevalence of chronic renal failure $(78.6 \%$ vs. $54.8 \%$; $\mathrm{P}<0.01)$ and had a trend towards acute coronary syndrome $(33.3 \%$ vs. $26.6 \% ; P=0.07)$. Type $B 2 / C$ target lesions were also more frequent in group $A(54.8 \%$ vs. $41.2 \%$; $P<0.01$ ), however, despite of these findings, these patients received less drug-eluting stents (18.1\% vs. $31.2 \%$; $P<0.01)$. There was no difference in mortality ( 0 vs $0.2 \% ; P=0.88$ ) or in the rate of procedure-related myocardial infarction (5.7\% vs. $3.3 \%$; $P=0.18)$. Emergency CABG was not required in any of the patients. Conclusions: Octogenarians, who currently account for about $20 \%$ of the patients treated by $\mathrm{PCl}$, had higher clinical and angiographic complexity, received less drug-eluting stents and had similar in-hospital results to the younger elderly individuals.

KEY-WORDS: Angioplasty. Drug-eluting stents. Coronary disease. Aged. Aged, 80 and over.

\footnotetext{
Instituto Dante Pazzanese de Cardiologia - São Paulo, SP, Brasil. Correspondência: Luiz Fernando Tanajura. Av. Dr. Dante Pazzanese, 500 - Vila Mariana - São Paulo, SP, Brasil - CEP 04012-909 E-mail: Iftanajura@uol.com.br

Recebido em: 5/9/2011 • Aceito em: 22/11/2011
} 
$\mathrm{H}$ á alguns anos a população mundial vivencia um processo de aumento da expectativa de vida, como pode ser observado em recente pesquisa em nosso meio realizada pelo Instituto Brasileiro de Geografia e Estatística, em meados da década passada, segundo a qual a média de idade dos brasileiros chegou a 71,3 anos, representando um crescimento de 0,8 ano em relação ao observado no início daquele período. ${ }^{1}$ Em consequência, tem havido aumento substancial da procura de serviços médicos por parte dessa população, caracterizada por doença coronária mais avançada e por mais comorbidades clinicamente relevantes. ${ }^{2} \mathrm{Q}$ uase que simultaneamente, ocorreu uma verdadeira revolução tecnológica nos materiais utilizados na cardiologia intervencionista, em especial pela introdução e posterior desenvolvimento dos stents farmacológicos, possibilitando seu uso com maior efetividade em pacientes com coronariopatia mais complexa. Dessa forma, pressupõe-se que tenham ocorrido mudanças significantes no afluxo e nas características de base de idosos encaminhados para a realização de intervenção coronária percutânea (ICP) nos últimos anos, pela eventual maior complexidade clínica dos casos tratados, a qual, possivelmente, se agrave com o aumento da faixa etária dos pacientes. ${ }^{3-5}$

O objetivo desta investigação foi comparar as características clínicas de base, os dados do cateterismo diagnóstico/terapêutico e os resultados hospitalares das ICPs realizadas em duas populações distintas de idosos (com idade $\geq 80$ anos e $<80$ anos) tratados contemporaneamente no Instituto Dante Pazzanese de Cardiologia (São Paulo, SP).

\section{MÉTODO}

No triênio 2008-2010, 998 pacientes com idade $>70$ anos foram tratados por meio de ICP em nossa instituição, correspondendo a $20,8 \%$ dos 4.799 casos tratados nesse período. De acordo com a proposta do estudo, foram divididos em dois grupos: grupo A, composto pelos 192 casos $(19,2 \%)$ com idade $\geq 80$ anos; e grupo B, constituído pelos $806(71,8 \%)$ pacientes restantes com idade $<80$ anos. Todos foram identificados a partir de um banco de dados informatizado, no qual foram incluídos de forma consecutiva e prospectiva. A natureza do estudo foi observacional. Foram incluídos pacientes com quadros clínico e angiográfico com indicação para ICP, de acordo com as diretrizes vigentes ${ }^{6}$, e que não apresentassem contraindicações ao uso da terapia antiplaquetária dupla.

As ICPs foram realizadas pela técnica convencional de liberação ótima, com implante direto ou pré-dilatação, o que era deixado a critério do cardiologista intervencionista responsável pelo procedimento. Os modelos de stents utilizados eram escolhidos de acordo com as características de cada caso; quando disponíveis, os stents farmacológicos eram reservados, em geral, para as situações de maior predisposição à reestenose.
0 esquema farmacológico adjunto consistiu de: 1) heparina não-fracionada, na dose de $100 \mathrm{Ul} / \mathrm{kg}$ de peso, administrada imediatamente antes do procedimento (ou $70 \mathrm{UI} / \mathrm{kg}$ de peso, quando administrada conjuntamente com os inibidores da glicoproteína IIb/llla); 2) ácido acetilsalicílico, na dose de ataque de $300 \mathrm{mg}$, seguida da dose de manutenção de $100 \mathrm{mg} / \mathrm{dia}$ (nos pacientes em uso crônico era mantida a dose de $100 \mathrm{mg} / \mathrm{dia}$, indefinidamente); 3) clopidogrel, na dose de ataque de $300 \mathrm{mg}$ (ou $600 \mathrm{mg}$, dependendo da forma clínica de apresentação da doença), seguida por $75 \mathrm{mg} / \mathrm{dia}$ ou ticlopidina (500 mg/dia), ambas com pré-tratamento realizado, nos casos eletivos, em tempo hábil (três dias para ticlopidina e um dia para clopidogrel). A duração do esquema antiplaquetário duplo foi de um mês para os pacientes submetidos ao implante de stents não-farmacológicos e de 12 meses para os que receberam modelos com liberação de medicamentos. Inibidores da glicoproteína IIb/llla poderiam ser prescritos nas doses habituais, sendo sua indicação deixada a critério do médico responsável pela intervenção. N enhum paciente recebeu os novos agentes antiplaquetários prasugrel ou ticagrelor.

Foram consideradas significativas lesões com diâmetro de estenose $>50 \%$, pelo critério visual. Além da presença de ao menos uma estenose significante e adequada para ICP, a indicação do procedimento sempre exigia a presença de isquemia miocárdica, expressa por sintomas ou alterações nos testes funcionais. As lesões-alvo foram classificadas de acordo com a proposta da força-tarefa americana. ${ }^{7}$ A função ventricular esquerda foi avaliada pelo critério visual. A angiografia coronária quantitativa não foi realizada de forma rotineira, o mesmo acontecendo com o ultrassom intracoronário.

\section{Definições}

Considerou-se sucesso do procedimento a obtenção de lesão residual inferior a $20 \%$, associada à ausência de complicações maiores (óbito, infarto do miocárdio ou cirurgia de revascularização miocárdica de urgência) durante a hospitalização índice. Infarto do miocárdio foi caracterizado pela presença de novas ondas $\mathrm{Q}$ no eletrocardiograma de 12 derivações e/ou por el evações dos marcadores bioquímicos de necrose (creatina quinase e sua fração MB), para níveis acima de três vezes do valor de referência. Insuficiência renal crônica foi definida como clearance de creatinina $<60 \mathrm{ml} / \mathrm{min}$, valor a partir do qual os pacientes eram triados para hidratação com soro fisiológico a 0,9\% ( $1 \mathrm{ml} / \mathrm{kg} / \mathrm{hora}$ ) pelo menos 6 horas antes da ICP e mantida 12 horas após. Doença coronária multiarterial foi definida com a observação de lesões coronárias $>50 \%$ em pelo menos dois dos vasos principais e/ou em seus ramos.

As variáveis quantitativas foram expressas em médias e desvio padrão e comparadas com a utilização do teste $t$ de Student. As variáveis qualitativas foram expressas 
por porcentagens e comparadas pelos testes qui-quadrado ou exato de Fisher, quando apropriado. Consideraram-se significantes valores de $P<0,05$, sendo todos os testes bicaudais.

\section{RESULTADOS}

A Tabela 1 discrimina as principais características clínicas de base, igualmente distribuídas entre os grupos, com exceção da média das idades, que, pela própria proposta do estudo, foi maior nos octogenários, e da presença de insuficiência renal crônica, mais comum no grupo A. O paciente mais idoso tratado contabilizava 99 anos de idade. Houve tendência de os mais idosos apresentarem-se mais frequentemente sob a forma de uma síndrome coronária aguda.

Quanto ao perfil angiográfico (Tabela 2), observou-se predomínio significativo das lesões de maior complexidade no grupo A, o mesmo ocorrendo com as intervenções na artéria descendente anterior. Lesões localizadas em enxertos venosos e no tronco da coronária esquerda foram tratadas de forma esporádica. ICP multiarterial $(17,7 \%$ vs. $16,7 \%$; $P=0,83)$ e obtenção de revascularização completa anatômica $(61,4 \%$ vs. $67,8 \% ; P=0,1)$ não diferiram, o mesmo acontecendo com o implante de stents com diâmetro inferior a $3 \mathrm{~mm}$ $(27,5 \%$ vs. $29,3 \% ; P=0,63)$ e com os modelos com extensão superior a 24 mm $(29,9 \%$ vs. 33\%; P = 0,38). O s stents farmacológicos foram menos frequentemente implantados nos pacientes mais idosos $(18,1 \%$ vs. $31,2 \% ; P<0,01)$.

Em relação aos eventos cardiovasculares na fase hospitalar, não foi encontrada diferença na mortalidade $(0$ vs. $0,2 \%$; $P=0,83)$ ou na ocorrência de infarto do miocárdio relacionado ao procedimento $(5,7 \%$ vs.
$3,3 \% ; P=0,18)$. Não houve necessidade da realização de cirurgia de revascularização miocárdica de urgência em nenhum paciente.

\section{DISCUSSÃO}

Este estudo comprovou que, de fato, ocorre atualmente expressivo afluxo de pacientes idosos para serem revascularizados por meio de $I C P$, uma vez que no triênio 2008-2010 um em cada cinco casos tratados apresentava 70 anos de idade ou mais; adicionalmente, também se aferiu que os octogenários correspondem a cerca de $20 \%$ dos pacientes revascularizados por via percutânea, sendo interessante notar que o paciente mais idoso dessa coorte apresentava idade quase centenária. Também nos parece relevante mencionar que este resultado corresponde ao dobro do relatado recentemente por um estudo de coorte europeu. ${ }^{2}$

Até o final da década retrasada havia questionamentos quanto à indicação de ICP em idosos, dúvida ainda mais pertinente nos mais idosos. No entanto, ensaios clínicos realizados na vigência de insuficiência coronária aguda e crônica deram respaldo à indicação de ICP nessas populações, justificando a expectativa de aumento do número de casos tratados. ${ }^{8-12}$

Em relação às características clínicas de base, observou-se maior prevalência de disfunção renal crônica nos octogenários, ratificando achados de estudos anteriormente publicados. ${ }^{9,12}$ Como os porcentuais de hipertensão arterial sistêmica e diabetes não apresentaram diferenças, torna-se claro que a maior faixa etária foi o principal responsável por esse achado, associado a pior prognóstico tardio e a maiores dificuldades e riscos quando da indicação de exames, invasivos ou não, que utilizem contrastes iodados. ${ }^{12}$

TABELA 1

Perfil clínico

\begin{tabular}{lccc}
\hline & $\begin{array}{c}\text { G rupo A } \\
\text { (n = 192) }\end{array}$ & $\begin{array}{c}\text { G rupo B } \\
\text { (n= 806) }\end{array}$ & P \\
\hline Idade, anos & $84,6 \pm 2,8$ & $73,6 \pm 2,7$ & $<0,01$ \\
Sexo feminino, \% & 41,6 & 37,9 & 0,38 \\
Hipertensão arterial sistêmica, \% & 73,1 & 71,2 & 0,84 \\
Diabetes melito, \% & 27 & 30,1 & 0,45 \\
Dislipidemias, \% & 65,1 & 69,1 & 0,32 \\
Infarto agudo do miocárdio prévio, \% & 33,8 & 38,5 & 0,25 \\
Cirurgia de revascularização míocárdica prévia, \% & 16,1 & 12,5 & 0,22 \\
Acidente vascular cerebral prévio, \% & 2,6 & 4,9 & 0,22 \\
Insuficiência renal crônica, \% & 78,6 & 54,8 & $<0,01$ \\
Quadro clínico, \% & & & 0,07 \\
$\quad$ Angina estável & 66,7 & 73,4 & \\
Síndrome coronária aguda & 33,3 & 26,6 & \\
\hline
\end{tabular}

$\mathrm{n}=$ número de pacientes. 
TABELA 2

Perfil angiográfico e do procedimento

\begin{tabular}{lccc}
\hline & $\begin{array}{c}\text { G rupo A } \\
\text { (n = 192) }\end{array}$ & $\begin{array}{c}\text { G rupo B } \\
\text { (n = 806) }\end{array}$ & P \\
\hline Doença multiarterial, \% & 52 & 47,6 & 0,3 \\
Vasos tratados, \% & & & 0,03 \\
DA & 42,9 & 37,4 & \\
CD & 28,7 & 29,7 & \\
CX & 17,6 & 26,1 & \\
TCE & 3 & 1,4 & \\
Pontes de safena & 7,7 & 5,2 & $<0,01$ \\
Lesões B2/C, \% & 54,8 & 41,2 & 0,9 \\
Lesões reestenóticas, \% & 4,6 & 4,7 & 0,83 \\
Intervenção coronária percutânea multiarterial, \% & 17,7 & 16,7 & 0,1 \\
Revascularização completa, \% & 61,4 & 67,8 & 0,63 \\
Stents <3 mm, \% & 27,5 & 29,3 & 0,38 \\
Stents > 24 mm, \% & 29,9 & 33 & $<0,01$ \\
Stents farmacológicos, \% & 18,1 & 31,2 & 0,55 \\
Sucesso do procedimento, \% & 92,7 & 94,1 & \\
\hline
\end{tabular}

$C D=$ artéria coronária direita; $C x=$ artéria circunflexa; $D A=$ artéria descendente anterior; $\mathrm{n}=$ número de pacientes; $T C E=$ tronco de coronária esquerda.

Em relação às indicações de ICP, muitos cardiologistas ainda optam por atitudes mais expectantes nos mais idosos, a não ser em presença de situações de angina mais limitante ou síndrome coronária aguda. ${ }^{8-11}$ Esta observação ganha ainda maior respaldo se atentarmos ao desenho do protocolo do estudo normativo TIME $^{9}$, conduzido apenas em pacientes com > 75 anos de idade e que apresentassem angina estável limitante (graus III ou IV). N este ensaio, os casos alocados para a estratégia invasiva apresentaram reduções significantes de hospitalizações por doença coronária (18\% vs. $72 \% ; P<0,001)$, hospitalizações causadas pela necessidade de procedimentos de revascularização (10\% vs. $48 \%$; P < 0,001) e eventos cardíacos maiores (26\% vs. 64\%; $P<0,001$ ), além de expressiva meIhora da qualidade de vida. Não houve impacto na mortalidade quando avaliada de forma isolada. ${ }^{13} \mathrm{Em}$ um subgrupo predeterminado de 188 pacientes, demonstrou-se que essas vantagens ocorreram sem aumento das despesas médicas. ${ }^{10}$ Por fim, 0 relato de quatro anos de evolução clínica tardia ratificou a vantagem da estratégia de revascularizar esses pacientes, uma vez que os tratados no primeiro ano exibiram maior sobrevida livre de eventos tanto no grupo invasivo como no conservador. ${ }^{11}$

Ainda no contexto da forma clínica de apresentação da coronariopatia, neste estudo houve tendência a maior número de casos tratados na vigência de síndrome coronária aguda nos pacientes do grupo $A$, dado que reforça os comentários do parágrafo anterior. As observações dos dados da angiografia de base, na qual fo- ram tratadas significativamente mais lesões de maior complexidade (B2/C) nos octogenários, corroboram esse achado, pois esse subtipo de lesão-alvo é mais comumente observado em situações de instabilidade. ${ }^{7}$

O utra observação relevante foi que, nos mais idosos, foram implantados significativamente menos stents com liberação de medicamentos, sabidamente associados a melhores resultados tardios, o que pode ser explicado pelo receio de complicações hemorrágicas, predisponentes de maior mortalidade tardia quando de grande porte. ${ }^{13,14}$ Recentemente, um grande ensaio clínico que avaliou um novo agente antiplaquetário em pacientes tratados por meio de ICP em síndrome coronária aguda demonstrou que é justamente nas faixas etárias mais elevadas que ocorrem os maiores impactos dessas complicações, em especial se acompanhadas pela coexistência de baixo peso corpóreo. ${ }^{14}$ Muitos cardiologistas têm receio natural com o implante de stents farmacológicos nos mais idosos, porque quando se implanta um desses modelos o período de uso do esquema antiplaquetário duplo sempre é mais prolongado, sendo de um ano na quase totalidade dos casos. $^{13,14}$ Como na atualidade estão em andamento diversos ensaios clínicos que avaliam a redução desse período, no caso de resultados positivos haverá tendência de reversão dessa expectativa, o que trará benefícios relevantes para esses pacientes, pois os mais idosos, de acordo com nossos resultados, apresentam no mínimo dois preditores de reestenose: maiores porcentuais de disfunção renal crônica e lesões de maior complexidade. ${ }^{15,16}$ 
Por fim, observou-se que, independentemente da faixa etária, o sucesso do procedimento foi elevado e as complicações reduzidas, ratificando achados prévios. ${ }^{9,12}$

\section{Limitações do estudo}

São consideradas limitações do estudo: a análise foi retrospectiva, ou seja, trata-se de um estudo comparativo entre duas coortes de pacientes, com todas as restrições na análise dos resultados que isso acarreta; não foi realizado seguimento tardio dos pacientes, impossibilitando uma comparação mais precisa e completa dos resultados clínicos dos dois grupos; e pela limitação ao uso dos stents com liberação de medicamentos, os benefícios potenciais advindos do emprego desses modelos, padrão de referência na atualidade, não puderam ser precisamente avaliados.

\section{CONCLUSÕES}

Seguindo uma tendência universal, observou-se que atualmente um em cada cinco pacientes tratados por meio de ICP tem $\geq 70$ anos de idade. Os mais idosos exibiram perfil mais complexo, tanto do ponto de vista clínico como do angiográfico; porém, possivelmente pela maior predisposição aos fenômenos hemorrágicos, receberam menos stents farmacológicos, observação relevante e potencialmente capaz de influenciar o prognóstico tardio. Apesar do cenário mais complexo, a cuidadosa seleção desses pacientes para ICP é capaz de trazer resultados clínicos satisfatórios e semelhantes aos do grupo menos idoso.

\section{CONFLITO DE INTERESSES}

Os autores declaram não haver conflito de interesses relacionado a este manuscrito.

\section{REFERÊNCIAS}

1. Nasri F. O envelhecimento populacional no Brasil. Einstein. 2008;6 Supl 1:S4-6.

2. Antonsen $L$, Jensen $L O$, Thayssen $P$, Christiansen $E H$, Junker $A$, Tilsted $\mathrm{HH}$, et al. Comparison of outcomes of patients $>80$ years of age having percutaneous coronary intervention according to presentation (Stable vs Unstable Angina Pectoris/non-STSegment Elevation Myocardial Infarction vs ST-Segment Elevation Myocardial Infarction). Am J Cardiol. 2011;108(10):1395-400.

3. Abizaid A, Cesar FB, Silva EM, Feres F, Mattos LAP, Staico $R$, et al. Stents farmacológicos: novos fármacos. Rev Bras Cardiol Invasiva. 2004;12(2):96-101.

4. Grines CL. Off-label use of drug-eluting stents putting in perspective. J Am Coll Cardiol. 2008;51(6):615-7.
5. Groeneveld PW, Matta MA, Greenhut AP, Yang F. Drugeluting compared with bare-metal stents among elderly patients. J Am Coll Cardiol. 2008;51(21)2017-24.

6. Mattos LA, Lemos Neto PA, Rassi A Jr, Marin-N eto JA, Sousa AGMR, Devito FS, et al. Diretrizes da Sociedade Brasileira de Cardiologia - Intervenção Coronária Percutânea e Métodos Adjuntos Diagnósticos em Cardiologia Intervencionista (II Edição - 2008). Rev Bras Cardiol Invasiva. 2008;16(2 Supl 2):9-88.

7. King SB $3^{\text {rd }}$, Smith $S C$ Jr, Hirshfeld JW Jr, Jacobs AK, M orrison DA, Williams DO, et al. 2007 focused update of the ACC/ AHA/SCAI 2005 guideline update for percutaneous coronary intervention: a report of the American College of Cardiology/ American Heart Association Task Force on Practice guidelines. J Am Coll Cardiol. 2008;51(2):172-209.

8. Seto TB, Taira DA, Berezin R, Chauhan MS, Cutlip DE, Ho $\mathrm{KK}$, et al. Percutaneous coronary revascularization in elderly patients: impact on functional status and quality of life. Ann Intern Med. 2000;132(12):955-8.

9. Pfisterer $M$, Buser $P, O$ sswald F, Allemann U, Amann $W$, Angehrn W, et al. Outcome of elderly patients with chronic symptomatic coronary artery disease with an invasive vs optimized medical treatment strategy: one-year results of the randomized TIME trial. JAMA. 2003;289(9):1117-23.

10. Claude J, Schindler C, Kuster GM, Schwenkglenks M, Szucs $T$, Buser $P$, et al. Cost-effectiveness of invasive versus medical management of elderly patients with chronic symptomatic coronary artery disease. Findings of the randomized trial of invasive versus medical therapy in elderly patients with chronic angina (TIME). Eur Heart J. 2004;25(24):2195-203.

11. Pfisterer M. Long-term outcome in elderly patients with chronic angina managed invasively versus by optimized medical therapy: four-year follow-up of the randomized Trial of Invasive versus Medical therapy in Elderly patients (TIME). Circulation. 2004;110(10):1213-8.

12. Tsai TT, Messenger JC, Brennan JM, Patel UD, Dai D, Piana RN, et al. Safety and efficacy of drug-eluting stents in older patients with chronic kidney disease. J Am Coll Cardiol. 2011; 58(18):1859-69.

13. Doyle BJ, Rihal CS, Gastineau DA, Holmes DR Jr. Bleeding, blood transfusion, and increased mortality after percutaneous coronary intervention: implications for contemporary practice. J Am Coll Cardiol. 2009;53(22):2019-27.

14. Wiviott SD, Braunwald E, MCCabe CH, Montalescot G, Ruzyllo W, Gottlieb S, et al. Prasugrel versus clopidogrel in patients with acute coronary syndromes. N Engl J Med. 2007;357(20):2001-15.

15. Mercado N, Boersma E, Wijns W, Gersh BJ, Morillo CA, de Valk $V$, et al. Clinical and quantitative coronary angiographic predictors of coronary restenosis: a comparative analysis from the balloon-to-stent era. J Am Coll Cardiol. 2001;38(3): 645-52.

16. West NEJ, Ruygrok PN, Disco CMC, W ebster MWI, Lindeboom WK, O'Neill WW, et al. Clinical and angiographic predictors of restenosis after stent deployment in diabetic patients. Circulation. 2004;109(7):867-73. 